\title{
Correction: Photonic-dispersion neural networks for inverse scattering problems
}

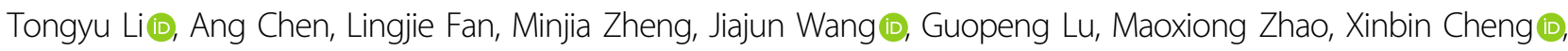
Wei Li, Xiaohan Liu, Haiwei Yin, Lei Shi (iD and Jian Zi (D)

Correction to: Light: Science \& Applications https://doi.org/10.1038/s41377-021-00600-y published online 27 July 2021

After publication of this article ${ }^{1}$, it is noticed this article contained an error. In Results section, the equation ' $C=|\Sigma| R d-R g \mid 2$ ' in the sentence below should be corrected to ' $C=\Sigma|R d-R g|^{2}$ '.

The difference between the generated response $R g$ and the detected response $R d$ (red block) is described with a cost function, for instance, mean square error $\mathrm{C}=\Sigma|R d-R g| 2$, reflecting in the fluctuations in parameter space. The original article has been updated.

Published online: 15 September 2021

\section{Reference}

Li, T., Chen, A. \& Fan, L. et al. Photonic-dispersion neural networks for inverse scattering problems. Light Sci. Appl. 10, 154 (2021). 